\title{
Molecular Ordering of 4-Acetyl-2'-Nitrobiphenyl - A Computational Analysis
}

\author{
Durga Prasad Ojha \\ Centre for Liquid Crystal Research and Education (CLCRE), Faculty of Physical Sciences, \\ Nagarjuna University, Nagarjuna Nagar (A.P.) - 522 510, India \\ Reprint requests to Dr. D. P. O.; Fax: 091-0863-235900
}

Z. Naturforsch. 55a, 918-922 (2000); received April 3, 2000

The molecular ordering of some biphenyl derivatives like 4-acetyl-2'-nitrobiphenyl (ANBP), 4-nitro2-biphenylamine (NBPA) and 4-acetyl-3'-chlorobiphenyl (ACBP) has been examined using basic concepts of quantum mechanics and intermolecular forces. The CNDO/2 method has been employed to compute the net atomic charge and atomic dipole components at each atomic centre. The modified Rayleigh-Schrödinger perturbation theory along with the multicentred-multipole expansion method has been employed to evaluate long-range interactions while a '6-exp' potential function has been assumed for short-range interactions. Accuracies upto $0.1 \AA$ in translation and $1^{\circ}$ in rotation have been achieved. Probability of occurrence of a particular configuration has been calculated using MB-statistics. On the basis of stacking and in-plane interaction energy calculations, all possible geometrical arrangements between molecular pairs have been considered and a most favorable configuration of pairing has been obtained. A comparative picture of molecular parameters like total energy, binding energy, total dipole moment etc. has been given. An attempt has been made to develop a molecular model correlated with the liquid crystalline property exhibited by this class of molecules.

Key words: Biphenyl Derivatives; CNDO/2 Method; Interaction Energy; MB-Statistics.

\section{Introduction}

The role of molecular forces and conformational properties in the mesomorphic behaviour has attracted the attention of a large number of workers [1-8]. Liquid crystal properties, such as the nematic-isotropic transition temperature and the entropy of transition, are influenced by the presence of alkyl chains. Semi-empirical calculations have been performed by several workers to examine from an energetic point of view the (i) internal distorsions and (ii) possibilities of motion in the aromatic core as well as in the terminal alkyl chains of mesomorphic compounds. Perrin and Berges have employed PCILO (Perturbational Configuration Interaction of Localized Orbitals) and INDO (Intermediate Neglect of Differential Overlap) methods to analse the influence of the conjugation between the oxygen lone pairs and the benzene ring on the internal rotations in several mesomorphic compounds. Further, it has been argued that a detailed analysis of pair interactions between the molecules of crystal lattices is expected to offer a better understanding of mesomorphism [9]. Tokita et al. [10] used a Lennard-Jones potential to evaluate intermolecular interactions between a couple of pure nematogens and attempted to correlate their results with the molecular field theory [11-13]. Inspired by the success of Tokita et al. [10], Sanyal et al. [14-16], using quantum mechanical methods such as CNDO (Complete Neglect of Differential Overlap) and perturbation techniques, have analysed various types of intermolecular interactions acting between sides, planes and terminals of a pair of molecules. It has been shown that the stability of molecular packing stemms is solely from dispersion forces, which is in agreement with the basic assumptions of molecular field theory $[11,13]$.

In the present paper, an attempt has been made to explain the behaviour of compounds using theoretical methods at varied angular and positional configurations in a molecular pair of 4-acetyl-2'-nitrobiphenyl (ANBP). The system chosen for the present investigation is not strictly liquid crystal line but seems to be quite interesting since it can be converted in to a liquid crystal by the choice of suitable substituents.

\section{Method of Calculation}

The net atomic charge and dipole moment components at each atomic centre have been computed using the $\mathrm{CNDO} / 2$ method [17]. Second order perturbation theo- 
ry for an intermediate range of interactions [18, 19] has been employed to evaluate pair interactions between the molecules of ANBP. The total interaction energy $\left(E_{\text {total }}\right)$ is expressed as

$$
E_{\mathrm{total}}=E_{\mathrm{el}}+E_{\mathrm{pol}}+E_{\mathrm{disp}}+E_{\mathrm{rep}}
$$

where $E_{\mathrm{el}}, E_{\mathrm{pol}}, E_{\mathrm{disp}}$, and $E_{\mathrm{rep}}$ represent electrostatic, polarization, dispersion and repulsion energy components, respectively. The energy values obtained from these computations were used to calculate the probability of each configuration using the Maxwell-Boltzmann formula [20]. The details of the formulae may be found in my earlier communications [21-24].

Energy minimization has been carried out separately for both, stacking and in-plane interactions. A detailed account of the interaction energy and probability distribution has been given for an intermediate distance $6 \AA$ for stacking and $8 \AA$ for in-plane interactions. Accuracies up to $0.1 \AA$ in translation and $1^{\circ}$ in rotation have been achieved. A selection of intermolecular separations has been made to allow the molecules to have full freedom corresponding to rotation and translation relative to each other. The origin has been chosen on an atom close to the centre of mass of the molecule, the $X$-axis along a bond parallel to the long molecular axis, the $Y$-axis in the plane of the molecules and the $Z$-axis perpendicular to the molecular plane. The molecular geometry has been constructed on the basis of the published crystallographic data [25].

\section{Results and Discussion}

The molecular geometry of ANBP is shown in Fig. 1 with various atomic index numbers. The net charge and dipole moment component corresponding to each of the atomic centres are listed in Table 1, while the total energy, binding energy and total dipole moment along with its components are given in Table 2. Table 3 represents a comparative picture of the total energy, binding energy and total dipole moments of ANBP with other systems like NBPA and ACBP (see Abstract). As evident from the Table 3, the total energy and binding energy of these molecules exhibit the following order:

$$
\begin{aligned}
& \mathrm{ANBP}>\mathrm{NBPA}>\mathrm{ACBP}, \\
& \mathrm{ANBP}>\mathrm{ACBP}>\mathrm{NBPA},
\end{aligned}
$$

while the dipole moments exhibt the order

$$
\mathrm{ACBP}>\mathrm{NBPA}>\mathrm{ANBP} \text {. }
$$

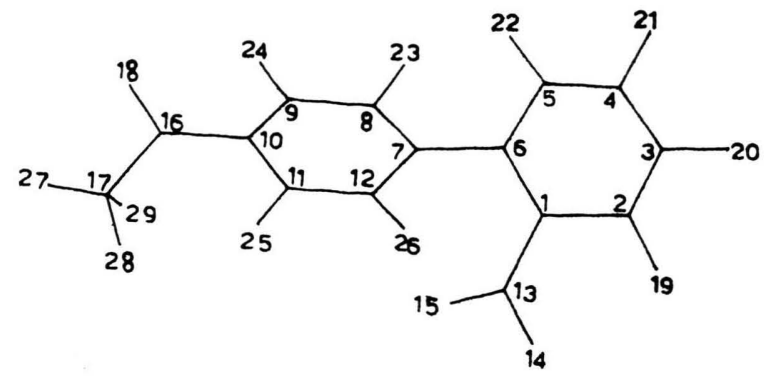

Fig. 1. Molecular geometry of the 4-Acetyl-2'-Nitrobiphenyl molecule with various atomic index numbers $(X$-axis along $1-2$

\begin{tabular}{|c|c|c|c|c|c|}
\hline \multirow[t]{2}{*}{ Sl. No. } & \multirow[t]{2}{*}{ Atom } & \multirow[t]{2}{*}{ Charge } & \multicolumn{3}{|c|}{ Atomic Dipole Components } \\
\hline & & & $X$ & $Y$ & $Z$ \\
\hline 1 & $\mathrm{C}$ & 0.030 & -0.113 & -0.213 & 0.069 \\
\hline 2 & C & 0.021 & -0.201 & 0.126 & 0.003 \\
\hline 3 & C & 0.011 & -0.177 & -0.052 & 0.019 \\
\hline 4 & C & 0.025 & -0.084 & -0.140 & -0.102 \\
\hline 5 & C & -0.005 & 0.059 & -0.165 & -0.089 \\
\hline 6 & C & 0.044 & 0.160 & -0.056 & -0.024 \\
\hline 7 & C & 0.049 & 0.053 & 0.014 & -0.010 \\
\hline 8 & $\mathrm{C}$ & -0.007 & 0.049 & -0.152 & -0.064 \\
\hline 9 & C & 0.029 & 0.066 & -0.147 & -0.057 \\
\hline 10 & $\mathrm{C}$ & -0.023 & 0.116 & 0.012 & 0.006 \\
\hline 11 & $\mathrm{C}$ & 0.019 & 0.073 & 0.088 & 0.076 \\
\hline 12 & C & -0.002 & -0.033 & 0.133 & 0.092 \\
\hline 13 & $\mathrm{~N}$ & 0.469 & -0.009 & -0.039 & 0.009 \\
\hline 14 & $\mathrm{O}$ & -0.339 & -0.497 & 1.014 & 0.837 \\
\hline 15 & $\mathrm{O}$ & -0.338 & 1.052 & 0.153 & -0.922 \\
\hline 16 & C & 0.248 & -0.064 & 0.116 & 0.089 \\
\hline 17 & C & -0.076 & 0.067 & 0.058 & 0.134 \\
\hline 18 & $\mathrm{O}$ & -0.265 & 0.557 & -1.029 & -0.654 \\
\hline 19 & $\mathrm{H}$ & 0.025 & 0.000 & 0.000 & 0.000 \\
\hline 20 & $\mathrm{H}$ & 0.004 & 0.000 & 0.000 & 0.000 \\
\hline 21 & $\mathrm{H}$ & 0.005 & 0.000 & 0.000 & 0.000 \\
\hline 22 & $\mathrm{H}$ & 0.002 & 0.000 & 0.000 & 0.000 \\
\hline 23 & $\mathrm{H}$ & -0.005 & 0.000 & 0.000 & 0.000 \\
\hline 24 & $\mathrm{H}$ & 0.004 & 0.000 & 0.000 & 0.000 \\
\hline 25 & $\mathrm{H}$ & -0.004 & 0.000 & 0.000 & 0.000 \\
\hline 26 & $\mathrm{H}$ & -0.002 & 0.000 & 0.000 & 0.000 \\
\hline 27 & $\mathrm{H}$ & 0.021 & 0.000 & 0.000 & 0.000 \\
\hline 28 & $\mathrm{H}$ & 0.028 & 0.000 & 0.000 & 0.000 \\
\hline 29 & $\mathrm{H}$ & 0.035 & 0.000 & 0.000 & 0.000 \\
\hline
\end{tabular}
bond).

Table 1. Calculated molecular charge distribution of 4-Acetyl2'-Nitrobiphenyl using the CNDO/2 method.

A study of the variation of the total stacking energy as a function of interplanar separation between two ANBP molecules corresponding to four distinct sets of rotations, viz. $X\left(0^{\circ}\right) Y\left(0^{\circ}\right), X\left(180^{\circ}\right) Y\left(0^{\circ}\right), X\left(0^{\circ}\right) Y\left(180^{\circ}\right), X\left(180^{\circ}\right)$ $Y\left(180^{\circ}\right)$ has been carried out, and it has been observed that the optimum interplanar separation between a pair of stacked ANBP molecules exclusively depends upon 
Table 2. Total energy ${ }^{\mathrm{a}}$, binding energy ${ }^{\mathrm{b}}$, total dipole moment and its components of the 4-Acetyl-2'-Nitrobiphenyl (ANBP) molecule. Total energy $=-174.74$ A.U., binding energy $=$ -16.08 A.U., total dipole moment $=2.62$ debyes.

\begin{tabular}{llrr}
\hline Components & $X$ & \multicolumn{1}{l}{$Y$} & \multicolumn{1}{l}{$Z$} \\
\hline Densities $^{+}$ & 1.13 & 1.35 & -0.55 \\
sp* $^{*}$ & 0.97 & -0.28 & -0.59 \\
pd $^{* *}$ & 0.00 & 0.00 & 0.00 \\
Total $^{++}$ & 2.09 & 1.07 & -1.14 \\
\hline
\end{tabular}

a The total energy corresponds to the sum of atomic as well as electronic energies of all constituents of the molecule in equilibrium geometry.

$\mathrm{b}$ The binding energy of a molecule is the difference between the total energy of the equilibrium molecular geometry and the sum of the atomic energies of the constituent atoms.

+ Contribution to dipole moment components due to electron densities.

$\mathrm{sp}^{*}$ hybridization moment. pd** hybridization moment.

++ Sum of electron density and hybridization contribution to dipole moment component.

Table 3. Total energy; binding energy; total dipole moment along with its components of ANBP, NBPA and ACBP molecules.

\begin{tabular}{llllllll}
\hline Molecule & $\begin{array}{l}\text { Total } \\
\text { Energy } \\
\end{array}$ & $\begin{array}{l}\text { Binding } \\
\text { Energy }\end{array}$ & \multicolumn{4}{l}{ Dipole Moment $(\mu)$ in Debyes } \\
\cline { 5 - 8 } & (A.U.) & $\mu_{X}$ & $\mu_{Y}$ & $\mu_{Z}$ & $\mu$ \\
\hline ANBP & -174.74 & -16.08 & 2.09 & 1.07 & -1.14 & 2.62 \\
NBPA & -153.09 & -14.40 & -3.15 & 0.33 & -0.46 & 3.20 \\
ACBP & -142.16 & -14.69 & -2.81 & 2.30 & 1.13 & 3.45 \\
\hline
\end{tabular}

the rotations given in one of the molecules about the $X$-axis and $Y$-axis with respect to the other. The complex corresponding to $X\left(0^{\circ}\right) Y\left(0^{\circ}\right)$ is more stable than the others. A deep and sharp minimum is observed for the rotational set of $X\left(0^{\circ}\right) Y\left(0^{\circ}\right)$, where the two molecules of ANBP exactly overlapp one can other with their planes separated by $6 \AA$. The variation of the probability with respect to rotation about the $Z$-axis is shown in Figure 2. The probabilities of various stacked complexes were evaluated at an interval of $10^{\circ}$ and show a sharp maximum at their minimum energy point. Having refined the interacting configuration with respect to translation along the $Z$-axis and rotation about the $Z$-axis at an equilibrium condition, the energy is brought down and the probability is further investigated with respect to translational motion in a stacked molecular pair along the $Y$-axis (Fig. 3), where it may be seen that the molecules in a stacked pair are capable of sliding along the $Y$-axis without much change of probability in a suitably good range.

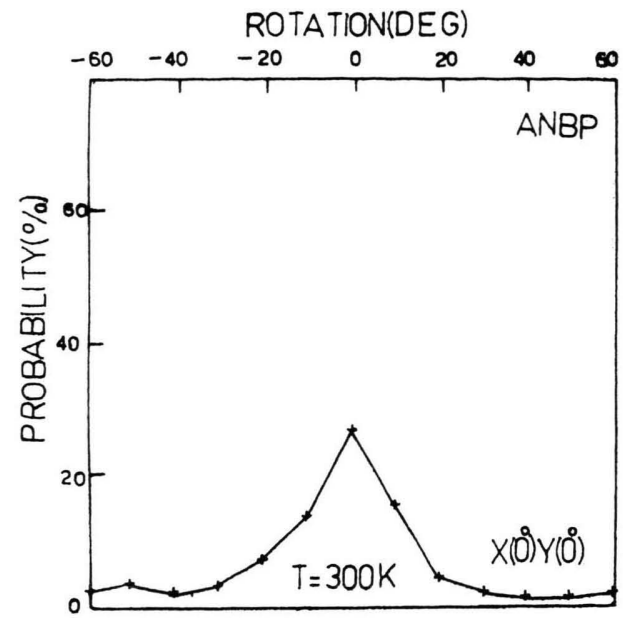

Fig. 2. Variation of the probability with respect to rotation about the $Z$-axis corresponding to the configuration indicated in the figure.

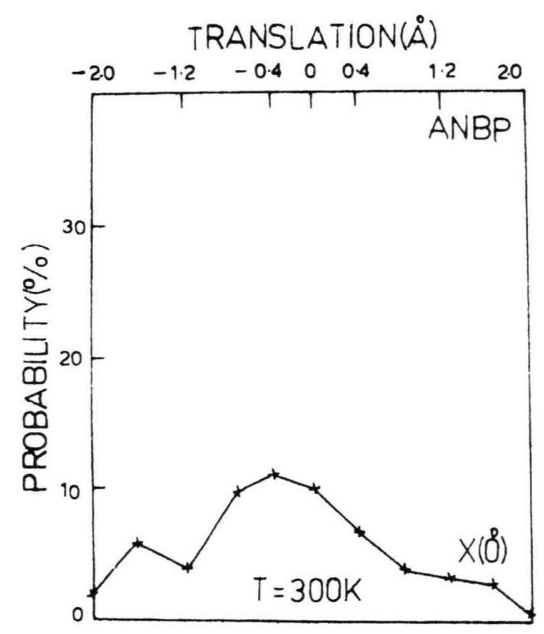

Fig. 3. Variation of the probability with respect to translation along the $Y$-axis during stacking interaction.

The variation of the in-plane interaction energy components with respect to translation along the long molecular axis ( $X$-axis) has been carried out corresponding to a particular rotation of $0^{\circ}$ about the $Y$-axis and the interaction energy is calculated by allowing translation in the range of $\pm 26 \AA$ at an interval of $2 \AA$. It has been observed that long-range forces are less significant than shortrange forces during in-plane interactions. Dispersion component is mainly responsible for the attractions between the pairs of ANBP molecules. Figure 4 shows 


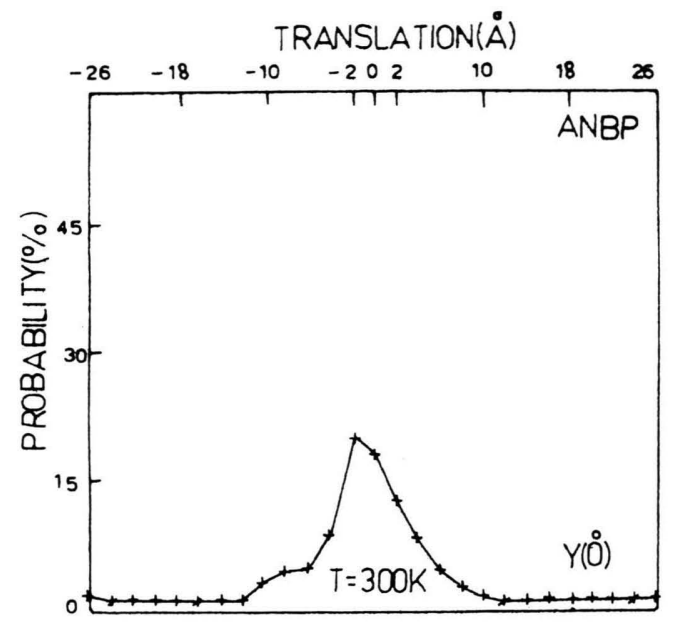

Fig. 4. Variation of the probability with respect to translation along the $X$-axis during in-plane interaction.

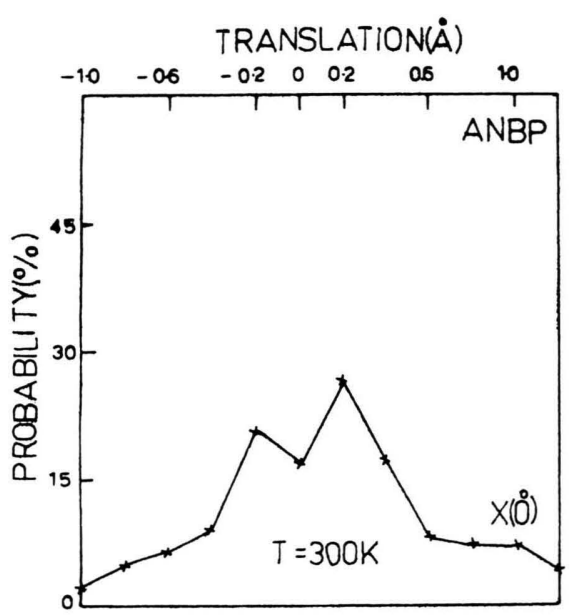

Fig. 5. Variation of the probability with respect to translation along the $Y$-axis during in-plane interaction.

the variation of the probability with respect to translation along the $X$-axis, while a similar analysis is presented in Fig. 5 for translation along the $Y$-axis. It may be observed that the molecule can be translated both along the $X$ - and $Y$-axis upto a suitable good range.

The energy corresponding to the optimum angle obtained initially has been further refined with an accuracies of $1^{\circ}$ in rotation and $0.1 \AA$ in translation. The final lowest energy stacked geometry is shown in Fig. $6 \mathrm{a}$ with an energy $-12.36 \mathrm{kcal} / \mathrm{mole}$. The in-plane minimum energy configuration is presented in Fig. 6b, which bears

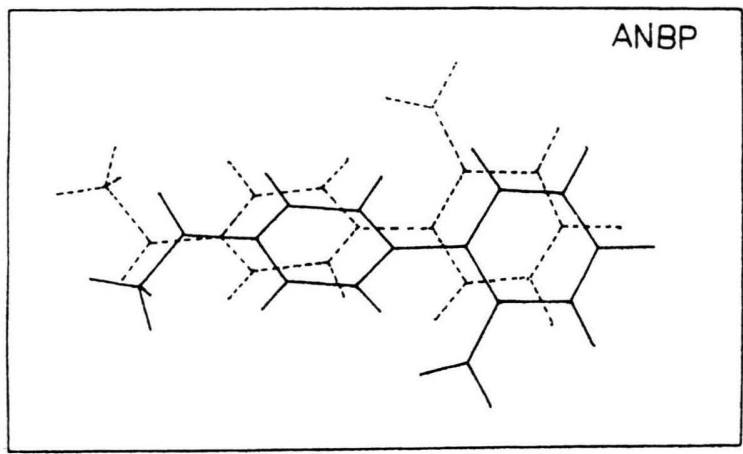

Fig. 6a. The lowest energy configuration obtained during stacking interaction with an energy $-12.36 \mathrm{kcal} / \mathrm{mole}$.

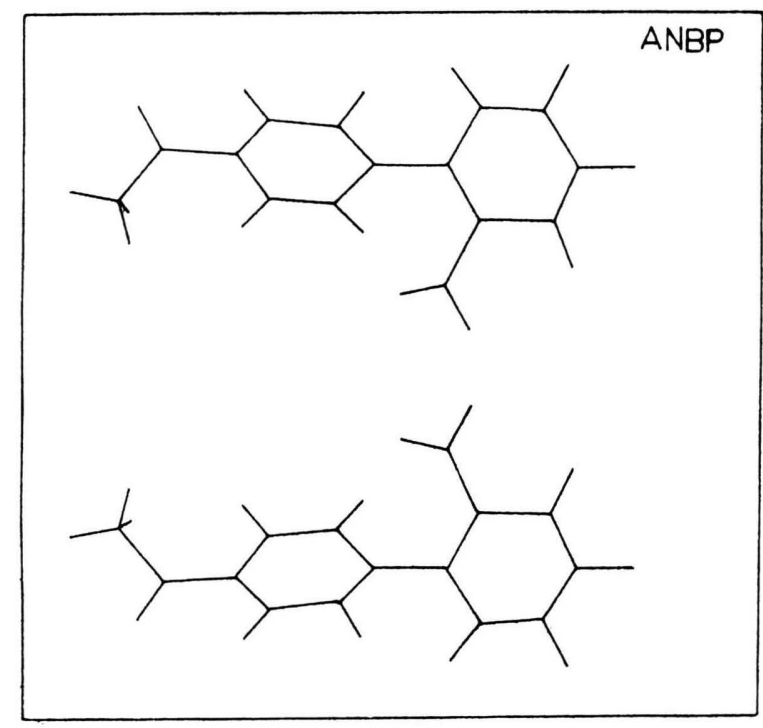

Fig. 6b. The lowest energy configuration obtained during inplane interaction with an energy $-7.56 \mathrm{kcal} / \mathrm{mole}$.

an energy $-7.56 \mathrm{kcal} / \mathrm{mole}$. The details of stacking as well as in-plane energy are listed in Table 4 with all the contributory terms to enable comparison. The interaction energy calculations for all possible configurations show that in a close packed structure, dispersion forces play a key role in stabilizing both the stacking and in-plane interactions.

Further, it may be observed that the large interaction energy valve and minimum energy configuration with the long molecular axes almost parallel to one another suggests a strong tendency to maintain order and conse- 
Table 4. Minimum energy obtained during stacking and inplane interactions between a pair of ANBP molecules after refinement. Energy is expressed in $\mathrm{kcal} / \mathrm{mole}$.

\begin{tabular}{lcc}
\hline Energy term & Stacking energy & In-plane energy \\
\hline$E_{\mathrm{QQ}}$ & -0.251 & 0.318 \\
$E_{\mathrm{QMI}}$ & -2.156 & -1.089 \\
$E_{\mathrm{MIMI}}$ & -1.894 & -1.245 \\
$E_{\mathrm{cl}}$ & -4.301 & -2.015 \\
$E_{\mathrm{pol}}$ & -1.958 & -0.776 \\
$E_{\mathrm{disp}}$ & -10.704 & -5.915 \\
$E_{\mathrm{rep}}$ & 4.602 & 1.145 \\
$E_{\text {total }}$ & -12.361 & -7.561 \\
\hline
\end{tabular}

quent existence of liquid crystallinity of the molecule. Therefore, unlike stacking which has a large contribution due to dispersion forces, in-plane attractions require more thermal activation and hence account for the high melting point of the system. At the melting point, thermal agitation will overcome the bindings of ANBP molecules in the crystal and the system will pass directly to the isotropic melt. However, the inherent tendency of the

[1] F. Laupretre and L. Mounerie, Eur. Polym. J. 14, 415 (1978).

[2] D. Sy and M. Patak, J. Physique-Lett. 40, L-137 (1979).

[3] M. Cotrait, P. Marsan, M. Pesquer, and V. Colpilhac, J. Physique 43, 335 (1982).

[4] G. W. Gray, Molecular Structure and Properties of Liquid Crystals, Academic Press, New York 1962.

[5] S. Chandrasekhar, R. Shashidhar, and N. Tara, Mol. Cryst. Liq. Cryst. 10, 337 (1970).

[6] P. G. de-Genns, The Physics of Liquid Crystals, 2nd ed, Oxford University Press, Oxford 1975.

[7] H. Perrin and J. Berges, J. Mol. Structure 76, 299 (1981).

[8] H. Perrin and J. Berges, J. Phys. Lett. 43, 531 (1982).

[9] P. J. Photinos and A. Saupe, J. Phys. Paris, 40C3, 389 (1979).

[10] K. Tokita, K. Fujimura, S. Kondo, and M. Takeda, Mol. Cryst. Liq. Cryst. 64, 171 (1981).

[11] W. Maier and A. Saupe, Z. Naturforsch, 13a, 564 (1958).

[12] W. Maier and A. Saupe, Z. Naturforsch, 14a, 882 (1959).

[13] W. Maier and A. Saupe, Z. Naturforsch, 15a, 287 (1960).

[14] N. K. Sanyal, S. N. Tiwari, and M. Roychoudhury, Mol. Cryst. Liq. Cryst. 132, 81 (1986). molecule to retain order even after melting does not escape the notice. Hence, if a suitable functional group is attached to the molecule, so that the length to breath ratio is increased with the dispersion energy, the molecules will show a change in long-range order, transition temperature and other related liquid crystalline properties.

The present computational analysis suggests a strong intermolecular interaction energy between two ANBP molecules, and a specific minimum energy configuration determines the alignment of the molecules with respect to one another. This may be correlated with the liquid crystalline property exhibited by this class of molecules. Also, the effect of substitution and its thermal stability may be predicted to a reasonable extent.

\section{Acknowledgement}

The financial support rendered by the Department of Science \& Technology, New Delhi, India is gratefully acknowledged.

[15] N. K. Sanyal, S. N. Tiwari, and M. Roychoudhury, J. Phys. Soc. Japan 55, 1171 (1986).

[16] N. K. Sanyal, S. N. Tiwari, and M. Roychoudhury, Mol. Cryst. Liq. Cryst. 140, 179 (1986).

[17] J. A. Pople and D. L. Beveridge, Approximate Molecular Orbital Theory, McGraw Hill, New York 1970.

[18] J. Caillet and P. Claverie, Biopolymers 13, 601 (1974).

[19] J. Caillet and P. Claverie, Acta Cryst. A31, 448 (1975).

[20] R. C. Tolman, The Principles of Statistical Mechanics, Oxford University Press, Oxford 1938.

[21] M. Roychoudhury, D. P. Ojha, and N. K. Sanyal, Mol. Cryst. Liq. Cryst. 163, 189 (1988).

[22] M. Roychoudhury and D. P. Ojha, Mol. Cryst. Liq. Cryst. 213, 73 (1992).

[23] M. Roychoudhury, D. P. Ojha, and N. K. Sanyal, Ind. J. Pure \& Appl. Phys. 32, 440 (1994).

[24] D. P. Ojha, M. Roychoudhury, S. N. Tiwari, and N. K. Sanyal, Advances in Statistical Phys. of Solids and Liquids (ed. S. Prakash \& K. N. Pathak), Wiley Eastern Ltd., New York (1990), p. 360.

[25] H. H. Sutherland, J. H. Hogg, and D. J. Willium, Acta Cryst. B30, 1562 (1974). 